\title{
Identification and differentiation of Trichophyton rubrum clinical isolates using PCR-RFLP and RAPD methods
}

\author{
A. Hryncewicz-Gwóźdź • T. Jagielski • \\ A. Dobrowolska • J. C. Szepietowski • E. Baran
}

Received: 15 November 2010 / Accepted: 21 December 2010/Published online: 18 March 2011

(C) The Author(s) 2011. This article is published with open access at Springerlink.com

\begin{abstract}
Trichophyton rubrum represents the most frequently isolated causative agent of superficial dermatophyte infections. Several genotyping methods have recently been introduced to improve the delineation between pathogenic fungi at both the species and the strain levels. The purpose of this study was to apply selected DNA fingerprinting methods to the identification and strain discrimination of $T$. rubrum clinical isolates. Fifty-seven isolates from as many tinea patients were subjected to species identification by polymerase chain reaction restriction fragment length polymorphism (PCR-RFLP) analysis and strain differentiation using a randomly amplified polymorphic DNA (RAPD) method, with two primers designated 1 and 6 . Using PCR-RFLP, 55 of the isolates studied were confirmed to be T. rubrum. Among those, a total of 40 and five distinct profiles were obtained by RAPD with primers 1 and 6 , respectively. The combination of profiles from both RAPD assays resulted in 47 genotypes and an overall genotypic diversity rate of $85.4 \%$. A dendrogram analysis performed on the profiles generated by RAPD with primer 1 showed most of the isolates $(87.3 \%)$ to be genetically
\end{abstract}

A. Hryncewicz-Gwóźdź $(\bowtie) \cdot J$. C. Szepietowski · E. Baran Department of Dermatology, Venereology and Allergology, Wrocław Medical University,

Chałubińskiego 1,

50-368 Wrocław, Poland

e-mail: anhryn@derm.am.wroc.pl

\section{T. Jagielski}

Department of Applied Microbiology, Institute of Microbiology, Faculty of Biology, University of Warsaw,

Warsaw, Poland

A. Dobrowolska

Department of Genetics of Microorganisms, University of Lódź, Łódź, Poland related. PCR-RFLP serves as a rapid and reliable method for the identification of T. rubrum species, while the RAPD analysis is rather a disadvantageous tool for T. rubrum strain typing.

\section{Introduction}

Dermatophytes comprise a highly specialized group of pathogenic fungi that infect keratinized tissues (skin, hair, and nails) of humans and animals, resulting in dermatophytoses, also referred to as tinea infections. Taxonomically, dermatophytes are classified into three genera: Epidermophyton, Microsporum, and Trichophyton, with the latter being the most complex, containing more than 15 species and several different variants within the species $T$. mentagrophytes [1]. The most frequently observed dermatophyte species worldwide is T. rubrum, whose infections usually manifest as tinea pedis and tinea unguium (onychomycosis). The prevalence of T. rubrum as a causative agent of onychomycosis is particularly high and exceeds $90 \%$ in Europe [2].

The identification of T. rubrum by means of conventional laboratory methods may not always be easy or straightforward, since T. rubrum exhibits substantial phenotypic variability. Contrastingly, a high degree of homogeneity of the T. rubrum genome, as revealed by using several anonymous molecular markers, significantly impedes discrimination at the strain level [3, 4]. Yet, as new molecular typing methods are becoming increasingly available, species determination and strain typing are still being improved.

The aim of this study was to apply some of the recently devised DNA fingerprinting methods to the identification and strain differentiation of T. rubrum. 


\section{Materials and methods}

The study included 57 isolates of T. rubrum recovered from as many dermatological patients from Lower Silesia, Poland (40), Kraków, Poland (8), and Tübingen, Germany (9).

Clinically, onychomycosis showed the highest number of cases (40 patients; $70.2 \%$ of all patients), followed by tinea pedis $(12 ; 21 \%)$, tinea corporis $(2 ; 3.5 \%)$, tinea cruris $(2 ; 3.5 \%)$, and tinea manuum $(1 ; 1.8 \%)$.

The isolates were primarily identified as $T$. rubrum on the basis of their phenotypic characteristics, such as colony surface texture, reverse pigmentation, the ability of microand macroconidia formation, and urease activity, assessed by standard mycological procedures.

A rapid mini-preparation procedure was used to extract fungal genomic DNA [5]. One $\mu \mathrm{L}$ of purified DNA (ca. $20 \mathrm{ng}$ ) was used for each polymerase chain reaction (PCR) assay.

The identification of $T$. rubrum was achieved by restriction fragment length polymorphism (RFLP) analysis of the internal transcribed spacer (ITS) region of rDNA, as previously described [6]. The amplified products were digested with MvnI, Hinfl, and, where necessary, with $M v a \mathrm{I}$ restriction endonucleases (Roche), at a 2-h incubation at $37^{\circ} \mathrm{C}$. The resulting restriction fragments were separated electrophoretically on $8 \%$ polyacrylamide gels and visualized under UV light after ethidium bromide (Et-Br) staining.

Two primers, designated 1 and 6 , as devised by Baeza and Mendes-Giannini [7], were used in two separate randomly amplified polymorphic DNA (RAPD) assays. Amplification products were resolved by electrophoresis using $1.5 \%$ agarose gels and were photographed under UV light after Et-Br staining. The gel images were analyzed by the Gel Doc system and Quantity One (Bio-Rad, USA) software. A dendrogram of the RAPD profiles obtained with primer 1 was constructed using Dice's coefficient of similarity and the unweighted pair-group arithmetic averaging (UPGMA) clustering method. An $80 \%$ genetic relatedness cutoff was used to define clusters.

\section{Results}

Of the 57 clinical isolates assessed in this study and recognized as $T$. rubrum by conventional identification methods, $55(96.5 \%)$ were further confirmed as T. rubrum by means of PCR-RFLP analysis. Using MvnI, all of the isolates except for one $(841 / 05)$ yielded restriction patterns consistent with that of Trichophyton taxon. Using Hinfl, 55 $(96.5 \%)$ isolates produced fragments whose sizes coincided with those expected for T. rubrum. One isolate (899/05) generated a pattern corresponding to either T. mentagro- phytes var. interdigitale or $T$. tonsurans. The isolate was eventually classified as T. tonsurans, based upon MvaI restriction analysis. One isolate $(841 / 05)$ could not be assigned to any of the dermatophyte species, distinguishable by their unique RFLP profiles obtained with MvnI, Hinfl, or MvaI enzymes.

Among the 55 T. rubrum isolates, a total of 40 distinct patterns (A-AN) were obtained by RAPD with primer 1 . Two additional patterns were observed for non-T. rubrum isolates (designated as BB and CC for isolate nos. 841/05 and 899/05, respectively). The four most prevalent patterns, designated as $\mathrm{T}, \mathrm{AJ}, \mathrm{AE}$, and $\mathrm{AN}$, contained five, four, three, and three isolates, respectively. Four patterns $(\mathrm{C}, \mathrm{AF}$, $\mathrm{AG}$, and $\mathrm{AL}$ ) contained two isolates each, and the remaining 34 patterns were represented by single isolates. The RAPD with primer 6 generated five different profiles in T. rubrum isolates (a-e) and two additional profiles in non-T. rubrum isolates (designated as "f" and "g" for isolate nos. 841/05 and 899/05, respectively). Among the $T$. rubrum isolates, the most abundant RAPD profile was that designated as " $b$ ", found in 23 isolates, followed by profiles designated as "a" and "c", represented by 19 and 11 isolates, respectively. Patterns designated as "d" and "e" were each identified for one isolate.

The combination of the profiles obtained with both primers resulted in 47 different genotypes for the T. rubrum isolates. Six of those genotypes were common to three (type T-a and type AJ-a) or two (types C-a, AE-b, AF-b, and $\mathrm{AN}-\mathrm{a}$ ) isolates, whereas the remaining 41 genotypes were unique, which are represented by single isolates only.

The distribution of the genotypes varied among the three geographical regions from which the isolates originated. Of the 40 RAPD genotypes produced with primer 1, each was restricted to only one geographical location. Twenty-six genotypes occurred in isolates from Lower Silesia, eight genotypes were observed in isolates from Kraków, and six in isolates from Tübingen, Germany. Regarding the genotypes obtained by RAPD with primer 6 , genotypes " $b$ " and "c" were found in isolates from all three regions studied, and genotype "a" was observed in the Lower Silesia and Tübingen isolates, but not in the Kraków isolates. The genotypes "a", "b", and "c" were the most prevalent in single regions, i.e., in Lower Silesia (44.7\% of isolates), Kraków (62.5\%), and Tübingen (44.4\%), respectively.

A dendrogram based on the RAPD profiles with primer 1 allowed the separation of the T. rubrum isolates into genetic similarity groups (clusters). A total of 48 isolates could be allocated into six main clusters (I-VI), with the similarity index between the isolates within the cluster being $80 \%$ or higher (Fig. 1). The largest cluster (VI) comprised 21 isolates, for which 11 different patterns were obtained. Clusters IV and V consisted of eight isolates each, 
Fig. 1 Dendrogram showing genetic relationships among 55 Trichophyton rubrum strains

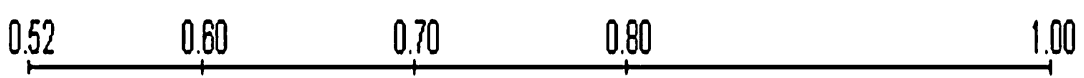
inferred from the randomly amplified polymorphic DNA (RAPD) patterns generated by using primer 1

being representative of either four (cluster IV) or eight (cluster V) distinct patterns. The numbers of isolates (and corresponding patterns) belonging to the remaining clusters were 3 ( 2 patterns) for cluster I, 5 (5) for cluster II, and 3 (3) for cluster III.

\section{Discussion}

The traditional, culture-based methods of identifying dermatophytes are cumbersome, laborious, and often inconclusive, due to fungal phenotypic variability and pleomorphism [1, 8]. However, the advent of molecular biology tools has enabled the development of new molecular approaches to the diagnosis of dermatophyte infections. One such approach, introduced by Jackson et al., relies upon RFLP analysis of PCR-amplified ITS regions of the rDNA gene complex [9]. This PCR-RFLP strategy was also employed in the present study. The only modification to the original procedure was the use of $M v n \mathrm{I}$ and Hinf $\mathrm{I}$ instead of MvaI. Whereas digestion with $M v n I$ allows discrimination between the three main dermatophyte genera (Trichophyton, Microsporum, and Epidermophyton) [10], the Hinfl digestion results in species-specific restriction profiles [11]. By using those two restriction enzymes, we wanted to verify their usefulness in the molecular identification of dermatophyte species, and T. rubrum in particular. The results from this study showed high concordance between conventional and molecular techniques for identifying $T$. rubrum. Only two isolates were identified as non- $T$. 
rubrum isolates: one was identified as T. tonsurans (upon restriction analysis with $M v a \mathrm{I}$ ) and the other was concluded to be a non-dermatophyte fungus. A possible explanation for the latter may relate to the laboratory contamination of the specimen and/or culture. It is also noteworthy that the application of the PCR-RFLP analysis, as in this study, does not allow to distinguish between T. rubrum and $T$. soudanense [9]. The distinction between the two species is only possible with methods that target single nucleotide polymorphisms (SNPs) within the ITS regions of rDNA [12]. Given, however, that T. rubrum differs substantially from $T$. soudanense in terms of phenotypic properties and geographical distribution (the former is cosmopolitan, while the latter is restricted mainly to the sub-Saharan part of Africa), the assignment to the T. rubrum species provided by PCR-RFLP seems unambiguous.

Differentiation of T. rubrum at the strain level has been attempted using a number of genotyping methods, though with unsatisfactory results $[3,13,14]$. However, Jackson et al. have recently reported on intraspecific variability within T. rubrum by PCR-amplifying two tandemly repetitive subelements (TRSs), located in the non-transcribed spacer (NTS) region of the rRNA gene cluster [15]. More recently, the RAPD analysis performed by Baeza et al. with two decameric primers, designated 1 and 6 , has been shown to produce a high degree of interstrain polymorphism $[7,16]$. The RAPD analysis with primers 1 and 6 was also applied in the present study, and this choice was motivated by a high discriminatory potential of the method, higher than that of the TRS typing system [16]. The RAPD typing demonstrated a high genetic diversity of the T. rubrum population studied. Based on the combined RAPD profiles, a total of 47 distinct genotypes were obtained. Hence, the overall genetic diversity rate (GDR), calculated as the number of different genotypes divided by the number of isolates, was $85.4 \%$. It is noteworthy that most of the polymorphism was generated by RAPD with primer 1 . It yielded 40 profiles, whereas RAPD with primer 6 resulted in only five profiles (GDRs of $72.7 \%$ and $9.1 \%$, respectively). In the first study that used primers 1 and 6 , among ten clinical isolates of T. rubrum, five molecular patterns were observed for each primer [7]. In a subsequent study including $67 \mathrm{~T}$. rubrum isolates, a total of 12 and 11 individual patterns were obtained by RAPD with primers 1 and 6 , respectively (GDRs of $17.9 \%$ and $16.4 \%$, respectively) [16]. Another study that investigated the intraspecific diversity of T. rubrum isolates originating from Japan and China revealed a considerable tightness of the population structure. All 150 isolates tested were split into only 19 fingerprinting genotypes, based on the combined RAPD analyses with primers 1 and 6 (GDR of 12.7\%) [17]. Much higher genotypic variability with the same primers was shown in a recent study of Santos et al. [18]. Nineteen different molecular profiles were configured for 52 T. rubrum isolates when each of the primers was used independently, resulting in a GDR of $36.5 \%$. It was speculated by the authors that the greater genetic diversity revealed in their study might result from having used strains exclusively from patients with onychomycosis. This condition, with its frequent chronicity, has been associated with mixed infections by multiple T. rubrum genotypes [19]. Collectively, the results of the studies cited above differ in terms of genetic polymorphism achieved by RAPD with both of the primers. The polymorphism obtained in our study was exceptionally high, and this may relate to the specific, genetic structure of Polish (and German) isolates, which is different from that of the so-far-analyzed T. rubrum populations.

The polymorphism obtained by RAPD with primer 1 was further investigated by a dendrogram analysis derived from the similarity coefficients between the RAPD patterns. Although the similarity coefficient value for the entire $T$. rubrum population studied was calculated to be $52 \%$, a vast majority of isolates $(87.3 \%)$ were distributed into six clusters, within which all of the isolates shared at least $80 \%$ similarity. This finding, considering that the similarity coefficient value of $0.8-0.99$ is assumed to represent genetically related isolates [20], indicates that the clustered isolates might have originated from the same strain that had undergone microevolutionary changes [16]. Thus, the high polymorphism resolved by RAPD may relate to the reproducibility of the RAPD technique itself. Indeed, RAPD assays often suffer from poor reproducibility and variations in the amplification patterns between isolates may be caused by even the slightest changes in the PCR reaction conditions. This explains the reluctance on the part of researchers to use the RAPD method and their search for newer molecular tools with a better diagnostic performance. One such promising alternative method is multilocus microsatellite typing (MLMT), which has recently been developed by Gräser et al. [4].

Finally, the fact that every genetic cluster identified in RAPD with primer 1 was restricted to a single geographical locale, together with marked differences in the frequencies of the three most prevalent genotypes produced from RAPD with primer 6 , may suggest that certain genotypes exhibit regional and/or geographical affinities. However, since this study was carried out on a relatively small sample of isolates, the geographical specificity of the genotypes would need to be confirmed by further research involving a larger population. Interestingly, the geographical differentiation of T. rubrum populations has, so far, been revealed only by using the MLMT methodology [4, 21].

In conclusion, the results from this study demonstrated the usefulness of PCR-RFLP in the rapid identification of T. rubrum, yet the insufficient suitability of the RAPD analysis for T. rubrum strain differentiation, due to its poor reproducibility. 
Acknowledgments The authors wish to thank Professor Martin Schaller (Mycological Laboratory, Universitäts-Hautklinik, Tübingen, Germany) and Professor Anna Macura (Mycological Department, Jagiellonian University, Kraków, Poland) for kindly providing the fungal strains.

Open Access This article is distributed under the terms of the Creative Commons Attribution Noncommercial License which permits any noncommercial use, distribution, and reproduction in any medium, provided the original author(s) and source are credited.

\section{References}

1. Weitzman I, Summerbell RC (1995) The dermatophytes. Clin Microbiol Rev 8:240-259

2. Tietz H-J, Kunzelmann V, Schönian G (1995) Changes in the fungal spectrum of dermatomycoses. Mycoses 38:33-39

3. Gräser Y, Kühnisch J, Presber W (1999) Molecular markers reveal exclusively clonal reproduction in Trichophyton rubrum. J Clin Microbiol 37:3713-3717

4. Gräser Y, Fröhlich J, Presber W, de Hoog S (2007) Microsatellite markers reveal geographic population differentiation in Trichophyton rubrum. J Med Microbiol 56:1058-1065

5. Liu D, Coloe S, Baird R, Pedersen J (2000) Rapid minipreparation of fungal DNA for PCR. J Clin Microbiol 38:471

6. White TJ, Burns T, Lee S, Taylor JW (1990) Amplification and direct sequencing of fungal ribosomal RNA genes for phylogenetics. In: Innis MA, Gelfand DH, Sninsky JJ, White TJ (eds) PCR protocols: a guide to methods and applications. Academic Press Inc., San Diego, CA, pp 315-322

7. Baeza LC, Mendes-Giannini MJS (2004) Strain differentiation of Trichophyton rubrum by random amplification of polymorphic DNA (RAPD). Rev Inst Med Trop Sao Paulo 46:339-341

8. Gräser Y, Kuijpers AF, Presber W, de Hoog GS (2000) Molecular taxonomy of the Trichophyton rubrum complex. J Clin Microbiol 38:3329-3336

9. Jackson CJ, Barton RC, Evans EG (1999) Species identification and strain differentiation of dermatophyte fungi by analysis of ribosomal-DNA intergenic spacer regions. J Clin Microbiol 37:931-936

10. Dobrowolska A, Stączek P, Kaszuba A, Kozłowska M (2006) PCR-RFLP analysis of the dermatophytes isolated from patients in Central Poland. J Dermatol Sci 42:71-74
11. Mochizuki T, Tanabe H, Kawasaki M, Ishizaki H, Jackson CJ (2003) Rapid identification of Trichophyton tonsurans by PCRRFLP analysis of ribosomal DNA regions. J Dermatol Sci 32:25-32

12. Kong F, Tong Z, Chen X, Sorrell T, Wang B, Wu Q, Ellis D, Chen S (2008) Rapid identification and differentiation of Trichophyton species, based on sequence polymorphisms of the ribosomal internal transcribed spacer regions, by rolling-circle amplification. J Clin Microbiol 46:1192-1199

13. Liu D, Coloe S, Pedersen J, Baird R (1996) Use of arbitrarily primed polymerase chain reaction to differentiate Trichophyton dermatophytes. FEMS Microbiol Lett 136:147-150

14. Zhong Z, Li R, Li D, Wang D (1997) Typing of common dermatophytes by random amplification of polymorphic DNA. Jpn J Med Mycol 38:239-246

15. Jackson CJ, Barton RC, Kelly SL, Evans EGV (2000) Strain identification of Trichophyton rubrum by specific amplification of subrepeat elements in the ribosomal DNA nontranscribed spacer. J Clin Microbiol 38:4527-4534

16. Baeza LC, Matsumoto MT, Almeida AM, Mendes-Giannini MJS (2006) Strain differentiation of Trichophyton rubrum by randomly amplified polymorphic DNA and analysis of rDNA nontranscribed spacer. J Med Microbiol 55:429-436

17. Yang X, Sugita T, Takashima M, Hiruma M, Li R, Sudo H, Ogawa H, Ikeda S (2009) Differentiation of Trichophyton rubrum clinical isolates from Japanese and Chinese patients by randomly amplified polymorphic DNA and DNA sequence analysis of the non-transcribed spacer region of the rRNA gene. J Dermatol Sci $54: 38-42$

18. Santos DA, Araújo RA, Hamdan JS, Cisalpino PS (2010) Trichophyton rubrum and Trichophyton interdigitale: genetic diversity among species and strains by random amplified polymorphic DNA method. Mycopathologia 169:247-255

19. de Assis Santos D, de Carvalho Araújo RA, Kohler LM, Machado-Pinto J, Hamdan JS, Cisalpino PS (2007) Molecular typing and antifungal susceptibility of Trichophyton rubrum isolates from patients with onychomycosis pre- and posttreatment. Int J Antimicrob Agents 29:563-569

20. Marol S, Yücesoy M (2008) Molecular epidemiology of Candida species isolated from clinical specimens of intensive care unit patients. Mycoses 51:40-49

21. Ohst T, de Hoog S, Presber W, Stavrakieva V, Gräser Y (2004) Origins of microsatellite diversity in the Trichophyton rubrum-T. violaceum clade (dermatophytes). J Clin Microbiol 42:4444-4448 